DFTT 15/96

April 1996

\title{
DISTRIBUTIONS IN FOUR-FERMION PROCESSES FOR W PHYSICS AT LEP 2
}

\author{
Elena ACCOMANDO, Alessandro BALLESTRERO \\ Giampiero PASSARINO
}

Dipartimento di Fisica Teorica, Università di Torino, Italy

INFN, Sezione di Torino, Italy

v. Giuria 1, 10125 Torino, Italy.

email:

accomando@to.infn.it, ballestrero@to.infn.it, giampiero@to.infn.it

The programs WPHACT and WTO, which are designed for computing cross sections and other relevant observables in the $e^{+} e^{-}$annihilation into four fermions, are used to make detailed and complete predictions for the semi-leptonic and fully hadronic channels $e^{+} e^{-} \rightarrow \bar{q} q l \nu, \bar{q} q \bar{q} q$. Both the total cross sections in the LEP 2 energy range and some of the most relevant distributions are analyzed. Particular algorithms are introduced for the fully hadronic channels in order to analyze the $W W$ physics and to properly define the signal versus the background. With appropriate kinematical cuts it has been shown that the Neutral Current background can be made vanishingly small when the problem of determining the $W$ boson mass is addressed. The remaining background from the complete Charge Current and Mixed processes is again small but not completely negligible. A detailed discussion is performed on the validity of the most relevant approximations such as the double-resonant one. The inclusion of final state QCD correction, in its naive form (NQCD), is discussed and various implementations are examined. 


\section{Introduction}

During the last year and in preparation for the experiments to be performed at LEP 2 [1], we have witnessed a new computational phase in the studies related to the process

$$
e^{+} e^{-} \rightarrow f_{1} \bar{f}_{2} f_{3} \bar{f}_{4} .
$$

If one neglects the fermion masses there are 32 distinct processes of this kind (classified in ref. [2]). Many of them have been studied at length in the literature, expecially for their interest in the physical properties of the $W$ boson [3] and of the Higgs boson [4], at energies ranging from that of Lep 1 up to $1 \mathrm{TeV}$ and above. Several groups have produced Fortran programs [2]-[5]-[6]-[0], whose results and predictions have been extensively compared during the LEP 2 Workshop [2]-[7]-[8]. These codes can be classified into three broad families, i.e. semi-analytical, deterministic and Monte Carlo (MC) integrators, including classical event generators (see ref.[2]). Some of them can produce accurate results for (almost) all four fermion processes, with the inclusion of all the relative Feynman diagrams which contribute and therefore well beyond some of the most popular and leading approximation such as the double resonant one.

While several different analyses can be performed with one of these programs we have concentrated in this paper on the theoretical predictions which are relevant for the physics of the $\mathrm{W}$ boson at Lep 2. In any study of this kind, one has to carefully consider both the theoretical uncertainties and the interplay between precision measurements and theoretical calculations.

Some of the published codes have reached an excellent technical agreement which is based on a certain choice of the input parameters, i.e. of the renormalization scheme, on the choice of the strategy for initial state radiations (structure functions, parton shower, YFS exponentiation as discussed in appendix A of ref. [9]), etc. This is highly satisfactory from a technical point of view, but the authors of the codes are fully aware of the fact that many approximations are still unavoidable at present. For instance initial and final state radiation are accounted for only at the level of the leading logarithms (few attempts to go beyond this approximation can be found in sect. 3.1.14 of ref. [2]), QCD corrections are taken into account in a naive way, which amounts to consider only some of the corrections to the vertices which become exact for a fully extrapolated setup, no electroweak corrections have been included so far, etc. This leads to a theoretical uncertainty which at present can only be roughly estimated by comparing some of the different options. A careful analysis on this point has been performed during the recent LEP 2 Workshop [2] and therefore it will not be repeated here.

As far as the interplay between precision measurements and theoretical calculations is concerned, let us first notice that typically a code will produce some differential cross section, including QED corrections and by using the exact matrix elements for the process, as a function of the center-of-mass energy and of the parameters of the vector bosons. It should be stressed at this point, that a semi-analytical code is by its own nature extremely precise but it allows at most cuts on two out of six of the final state invariant masses. However we have at our disposal codes which are not only using the exact matrix elements but also which allows for cuts on all other variables. A crucial role in this context is played by the hadronization process [10]. At least in first approximation we 
could say that the four-fermion codes will describe the electroweak content of the process to very high accuracy but they are lacking perturbative parton shower or non-perturbative hadronization. This raises the question of their reliability for the study of hadronic or mixed hadronic-leptonic final states. Even though a pragmatic solution, adopted by many codes, consists in standard interfacing with parton-shower and hadronization programs we still insist on the importance of presenting the most precise predictions for cross sections and distributions as they result from the dedicated codes with the inclusion of final states QCD perturbative corrections. It has been shown by the LEP 1 collaborations that such predictions are indeed of the upmost importance for understanding the underlying physical properties of the model once the proper de-convolution procedure is applied to the data. For this reason we are still thinking that a correct (theoretical) treatment of the problem at the level of exact and full matrix elements (including perturbative QCD corrections) will be essential in understanding several features, not least the quantitative effect of some of the most common approximations and the relevance of background versus signal, all of this in presence of some set of simple but realistic enough cuts.

As already mentioned, in this paper we will analyse the basic properties of the $W$ boson, having in mind expecially but not only the determination of the $W$ boson mass. It is our opinion that despite the numerous investigations and the various attempts no systematized effort has been spent so far in applying dedicated four-fermion codes to examine the problem in full detail. We have started from two codes, WPHACT and WTO [6], which are described at length in the literature and which are based on completely different methods and approaches. Not only the event generation is different in the two codes but also the full theoretical framework is based on non intersecting methods. The possibility of comparing results for various physical observables, all computed with very high numerical accuracy, gives us an almost absolute confidence on our results and allows us to give reliable predictions upon which different analyses and strategies can be based.

Our goal has been therefore to investigate all the Charge-Current processes (CC) (typically $\bar{u} d c \bar{s}$ ) which are relevant for a measurement of $M_{W}$ from $W W$ threshold cross sections and for a direct reconstruction of $M_{W}$. At the same time we have been able to perform a detailed study of the corresponding background induced by Neutral-Current processes (NC) (typically $\bar{u} u s \bar{s}$ ) and by the mixed ones (Mix) (typically $\bar{u} d u \bar{d}$ ). This is a highly non-trivial affair since the fully hadronic channel $e^{+} e^{-} \rightarrow \bar{q} q \bar{q} q$ will receive contributions from 7 different types of physical processes: one CC (11 diagrams), five NC (32 or 64 diagrams since we include gluons) and one Mix (43 diagrams when again gluons are included). We have not included in our analysis reducible backgrounds such as those due to four jet processes containing two gluons and two quark jets, which can hopefully be distinguished from four quark jets or evaluated with other dedicated codes as those used in ref. [11].

The outline of the paper is as follows. In Sect. 2 we briefly introduce the theoretical framework and explain the main conceptual differences between WPHACT and WTO. In Sect. 3 we concentrate on the semi-leptonic channel $e^{+} e^{-} \rightarrow \bar{q} q l \nu$ with particular emphasis on the final state electron. In Sect. 4 we discuss the fully hadronic channel. Sect. 5 is devoted to the conclusions. 


\section{Theoretical framework}

Ideally the object to be investigated is the cross section for $W W$ production which increases very rapidly near the nominal kinematic threshold. However we must deal with final states which contain four fermions in a situation where, for instance, there is no quark-tagging. Therefore a fully hadronic final state will include all possible combinations of quarks which is by far more complicated than simply analyzing $d \bar{u} c \bar{s}$ or similar ones. To understand the complexity of the problem we start by classifying all the relevant processes. First the semi-leptonic

- $\mu^{-} \bar{\nu}_{\mu} u \bar{d}(c \bar{s})\left[\mu^{+} \nu_{\mu} \bar{u} d(\bar{c} s)\right]$,

- $e^{-} \bar{\nu}_{e} u \bar{d}(c \bar{s})\left[e^{+} \nu_{e} \bar{u} d(\bar{c} s)\right]$,

next the fully hadronic ones

- $u \bar{d} s \bar{c}(d \bar{u} c \bar{s})$

- $u \bar{u} d \bar{d}(c \bar{c} s \bar{s})$,

- $u \bar{u} c \bar{c}$,

- $u \bar{u} s \bar{s}(u \bar{u} b \bar{b}, c \bar{c} d \bar{d}, c \bar{c} b \bar{b})$,

- $d \bar{d} s \bar{s}(d \bar{d} b \bar{b}, s \bar{s} b \bar{b})$.

- $u \bar{u} u \bar{u}(c \bar{c} c \bar{c})$.

- $d \bar{d} d \bar{d}(s \bar{s} s \bar{s}, b \bar{b} b \bar{b})$.

The leading contribution below the $Z Z$ threshold is given by those processes where the fermions can be paired in such a way that they can derive from a decaying $W$ (even if they do not from the point of view of Feynman diagrams). Thus for the fully hadronic channel we get dominant contributions from two processes, each counted with its own multiplicity,

$$
e^{+} e^{-} \rightarrow d \bar{u} c \bar{s}, \quad d \bar{u} u \bar{d}
$$

which we term signal while the rest will be referred as background. Strictly speaking even the signal receive some sort of contamination, since $d \bar{u} u \bar{d}$ has a part which comes from Neutral-Currents. Our terminology will be the standard one, therefore

- semi-leptonic processes with a $\mu$ are referred as CC10 processes,

- semi-leptonic processes with a $e$ are referred as CC20 processes,

- fully hadronic processes are referred as CC11 or NC32 or NC64 or Mix43. 
WPHACT and WTO can deal with all the above channels and for them they can produce all relevant distributions, from total cross sections to differential cross sections in energies, scattering angles, invariant masses or any of their combinations. Both codes can have kinematical cuts and for that we assumed as a starting point a commonly accepted choice, the so called canonical cuts which we briefly summarize:

- $E_{l} \geq 1 \mathrm{GeV}, E_{q} \geq 3 \mathrm{GeV}$.

- $M\left(q_{i}, q_{j}\right), M\left(q_{i}, \bar{q}_{j}\right), M\left(\bar{q}_{i}, \bar{q}_{j}\right) \geq 5 \mathrm{GeV}$.

- $10^{\circ} \leq \theta_{l} \leq 170^{\circ}$.

- $\theta\left(l_{i}, q_{j}\right), \theta\left(l_{i}, \bar{q}_{j}\right) \geq 5^{o}$.

In addition we have selected more restrictive cuts whenever this was of any relevance for the discussion.

The long write-up of WPHACT and of WTO [6] can be found in the literature and here we briefly summarize their main features.

For WPHACT the code for the full tree level matrix elements for each final state four fermion process has been written semi-automatically by means of a set of routines PHACT [12] ( Program for Helicity Amplitudes Calculations with Tau matrices ) which implements the helicity formalism of ref. [13]. Different phase spaces are employed to entertain the complex peaking structure of the Feynman diagrams. The adaptive routine VEGAS [14] is used for integrating over the phase space. All momenta are explicitly computed in terms of the integration variables and therefore any kinematical cut can be easily performed as well as distributions for any observable. WPHACT can be also used as a flat event generator.

For WTO the helicity amplitudes for each given process are given, according to the formalism of ref. [15], in terms of the 7 independent invariants which characterize the phase space. The phase space itself, including all realistic kinematical cuts, is also described in terms of invariants. The numerical integration, with complete cut-availability, is performed with the help of a deterministic integration routine which makes use of quasirandom, deterministic number sets, the shifted Korobov sets. The boundaries of the phase space, with kinematical cuts, are reconstructed through a backwards propagation of constraints.

For both codes, initial state QED radiation is included by means of the structure function approach and upon initialization the final state QCD corrections are included by adopting a naive approach (NQCD) to which we will come back later in this section.

There are external blocks present both in WPHACT and in WTO which, although with a different implementation, have a common root. Among them we will quote, as most relevant, the choice of the renormalization scheme and the question of final state QCD corrections.

Four-fermion physics is right now a tree-level prediction and therefore one can play with the available experimental data points. As an example we observe that one of the key relations among the parameters is 


$$
G_{\mu} M_{W}^{2}=\frac{\pi \alpha}{\sqrt{2} s_{W}^{2}} \frac{1}{1-\Delta r}
$$

We have indicated with $G_{\mu}$ the Fermi coupling constant, with $\alpha$ the fine structure constant and with $s_{W}$ the sinus of the weak-mixing angle. The definitions of the radiative correction factors $\Delta r, \Delta \rho, \Delta r_{r e m}$ are given in ref. [16]. Given the absence of a full one-loop calculation we have at our disposal essentially two non-pathological options, i.e. the so called $\alpha$ scheme

$$
\begin{aligned}
s_{W}^{2} & =\frac{\pi \alpha}{\sqrt{2} G_{\mu} M_{W}^{2}}, \quad \Delta \rho=\Delta r_{r e m}=0, \\
g^{2} & =\frac{4 \pi \alpha\left(2 M_{W}\right)}{s_{W}^{2}}
\end{aligned}
$$

$g$ being the $S U(2)_{L}$ coupling constant and the so called $G_{\mu}$-scheme

$$
\begin{aligned}
s_{W}^{2} & =1-\frac{M_{W}^{2}}{M_{Z}^{2}}, \\
g^{2} & =4 \sqrt{2} G_{\mu} M_{W}^{2}, \quad \Delta \rho=\Delta r_{r e m}=0,
\end{aligned}
$$

which is requested by Ward Identities of the theory. We decided to use the latter as our preferred set-up.

To explain our naive treatment of QCD (NQCD) we consider the CC10 process $e^{+} e^{-} \rightarrow$ $\mu^{-} \bar{\nu}_{\mu} u \bar{d}$. In general one would like to include final state QCD corrections, even when kinematical cuts are imposed, however a full calculation is missing. Thus we make use of naive QCD, a simple recipe where the total $W$-width is corrected by a factor

$$
\Gamma_{W} \rightarrow \Gamma_{W}\left(1+\frac{2}{3} \frac{\alpha_{s}\left(M_{W}\right)}{\pi}\right),
$$

where $\alpha_{s}$ is the strong coupling constant.The cross section gets multiplied by a naive factor even in the presence of cuts

$$
\sigma_{C C 10, C} \rightarrow \sigma_{C C 10, C}\left(1+\frac{\alpha_{s}\left(M_{W}\right)}{\pi}\right) .
$$

This naive approach, consequence of our ignorance about the complete result, would be correct only for $\sigma_{C C 03, e x}$, the double-resonant approximation with fully extrapolated setup. For $\sigma_{C C 10, C}$ it is instead only a rough approximation because of two reasons. First of all in $\mathrm{CC} 10$ we have not only a virtual QCD correction to the $W u \bar{d}$ vertex but also a box diagram. Moreover QED and QCD radiation are quite different if cuts are imposed, expecially in presence of severe cuts. Thus any inclusion of final state QCD corrections is, at present, only a very crude approximation which moreover can become quite bad whenever stringent kinematical cuts are applied to the process.

To clearly state the accuracy of our calculations we must add that QED radiation is included by means of the structure function approach (in the so-called $\beta$-scheme (see 
appendix A of ref. [9]). The effect of QED final state radiation must certainly be included for any reliable determination of the physical observables at LEP 2 but we have decided for not including it in the present analysis since a more detailed theoretical investigation is needed.

Before entering the details of our analysis we will give one example of the fine-tuning between WPHACT and WTO. Given the cross-section for a specific choice as $u \bar{d} s \bar{c}$ with canonical cuts we have reported in Fig. 1 the relative deviations from our weighted average as a function of $\sqrt{s}$. The emerging picture clearly illustrates that we have reached a very high level of technical agreement. Actually this figure will be the only one where we report separately results from WPHACT and WTO. For the rest of the paper our results should be interpreted as a common WPHACT/WTO calculation, which have differences well below what could eventually be appreciated in any realistic figure. As it will be discussed later there are different implementations of the naive QCD corrections in WPHACT and in WTO. Fig. 1a gives the comparison between the preferred setups of each code showing differences of order $\left(\alpha_{s} / \pi\right)^{2}$ whereas we have reported in Fig. 1b the tuned-NQCD comparison clearly showing that below $0.1 \%$ everything really matters.

The exact definition of a vector boson mass, to be extracted from some set of data is, to a large extent, dependent on the adopted scheme, i.e. constant or running width etc. Thus any calculation which aims to determine $M_{W}$ should clearly state the exact set of conventions under which it has been produced. For us a $W$-propagator of invariant mass $s$ is defined by

$$
\Delta_{W}^{-1}(s)=s-M_{W}^{2}+i \frac{s}{M_{W}} \Gamma_{W} .
$$

corresponding to the so-called running width scheme.

We now specify our set of input-parameters. In the actual calculations we used

$$
\begin{gathered}
M_{Z}=91.1884 \mathrm{GeV}, \quad M_{W}=80.26 \mathrm{GeV}, \\
\Gamma_{Z}=2.4974 \mathrm{GeV}, \quad \alpha^{-1}\left(2 M_{W}\right)=128.07 .
\end{gathered}
$$

In both codes the value of $\Gamma_{W}$ is derived within the minimal standard model. As for $\alpha\left(2 M_{W}\right)$ its value is only relevant for the Coulomb correction factor since otherwise we are working in the $G_{\mu}$-scheme. NQCD is implemented according to $\alpha_{s}\left(M_{Z}\right)=0.123$ (input) giving $\alpha_{s}\left(M_{W}\right)=0.1255$.

\section{Semi-leptonic channel $\bar{q} q l \nu$}

This channel, which is relatively easy and clean from a theoretical point of view, is characterized by the presence of two (or more) hadronic jets, an isolated energetic lepton and missing energy. We have not taken into account the $\tau$ as in this case one has a narrow jet due to the hadronic $\tau$ decays. Our cuts will require a threshold energy of $1 \mathrm{GeV}$ for the lepton, with a $10^{\circ}$ cuts with respect to the beams. Full angular coverage is required for the two quarks but their invariant mass has to be greater than $5 \mathrm{GeV}$. The lepton is also required to be isolated from the hadronic jets which in our set-up translate into an angular cuts of $5^{\circ}$. We have computed various quantities: 
- the total cross section as a function of $\sqrt{s}$, from $150 \mathrm{GeV}$ to $205 \mathrm{GeV}$ for various choices of the input parameter $M_{W}$.

- Several relevant distributions. For the semi-leptonic channel there is no ambiguity in defining $M_{+}$as it can be reconstructed by using $M(u \bar{d})$ both in $e^{+} e^{-} \rightarrow \mu^{-} \bar{\nu}_{\mu} u \bar{d}$ and in $e^{+} e^{-} \rightarrow e^{-} \bar{\nu}_{e} u \bar{d}$. Thus we have computed

$$
\frac{d \sigma}{d M_{+}}
$$

for a large interval of $M_{+}$and for $\sqrt{s}=161,175,190 \mathrm{GeV}$. The $\sqrt{s}=161 \mathrm{GeV}$ case has been added for completeness even though of little experimental interest.

The total cross section as a function of $\sqrt{s}$ is reported in Fig. 2 where we have included a corresponding weight of 4 which properly takes into account the following processes

$$
\begin{aligned}
& e^{+} e^{-} \rightarrow \mu^{-} \bar{\nu}_{\mu} u \bar{d}, \mu^{-} \bar{\nu}_{\mu} c \bar{s}, \mu^{+} \nu_{\mu} \bar{u} d, \mu^{+} \nu_{\mu} \bar{c} s \\
& e^{+} e^{-} \rightarrow e^{-} \bar{\nu}_{e} u \bar{d}, e^{-} \bar{\nu}_{e} c \bar{s}, e^{+} \nu_{e} \bar{u} d, e^{+} \nu_{e} \bar{c} s
\end{aligned}
$$

The high energy tail of the figure starts showing a minor difference between muons and electrons.

The technical agreement in our predictions enforces the confidence on $\sigma\left(E_{c m}, M_{W}\right)$ upon which one must rely for a 1(2)-point scan needed in the $M_{W}$ measurement.

In computing the cross section $\sigma\left(\sqrt{s}, M_{W}\right)$ we have payed particular attention to quantities which reflect the sensitivity to the $W$ mass. In particular we have examined and computed

$$
\sigma\left|\frac{d M}{d \sigma}\right|, \quad \sqrt{\sigma}\left|\frac{d M}{d \sigma}\right|, \quad\left|\frac{d M}{d \sigma}\right|,
$$

which contribute to the statistical error and to the systematic errors on $M_{W}$. All quantities are reported in Fig. 3 as a function of $\sqrt{s}-2 M_{W}$ with a nominal $W$ mass of $80.26 \mathrm{GeV}$. In agreement with previous findings we observe that the statistical sensitivity factor is essentially flat within $(\sqrt{s})_{\min } \pm 2 \mathrm{GeV}$ where it varies of approximately $.05 \mathrm{GeV} \mathrm{pb}{ }^{-1 / 2}$.

For completeness we have shown in Fig. 4 the total cross section for $e^{+} e^{-} \rightarrow e^{-} \bar{\nu}_{e} u \bar{d}$ for different values of $M_{W}$.

Actually in computing distributions we have been able to compare two rather different approaches. WPHACT usually collects all the data in a single run in which a binning procedure can be automatically started, just giving the variables for which distributions are to be evaluated and the corresponding binning. WTO instead avoids the binning (even though this procedure is implemented) and computes directly the differential cross section by integrating each time over an eight-fold phase space. As a result of the comparison of the two approaches, i.e. something that in principle is a fast procedure (WPHACT) compared with a slow but accurate one (WTO), we always obtain that fast is also accurate enough in all relevant regions. Thus we can state that the shape and the moments reconstructed from the distributions are in excellent agreement. From a purely technical 
point of view, it may be relevant to notice that the curves of the distributions reported in the figures were obtained from WPHACT with a number of bins ranging from 40 to more than 100 per curve. Moreover the statistical errors obtained from WTO and WPHACT are simply not visible in the plots.

In Fig. 5 we have shown $d \sigma / d M_{+}$where $M_{+}=M(u \bar{d})$ for $\sqrt{s}=161,175 \mathrm{GeV}$ and $190 \mathrm{GeV}$ and for $l=e$. There are no appreciable differences if we consider $l=\mu$, largely due to our kinematical cuts. We observe that the distribution becomes more and more symmetric around $M(u \bar{d})=M_{W}$ with growing $E_{c m}$. From the distributions we have reconstructed four quantities: the maximum $M_{\max }$, the mean $\langle M\rangle$ and the first moments $S_{2,3}$. They are reported in the following table

\begin{tabular}{|c|c|c|c|c|c|}
\hline Final state & $\sqrt{s} \mathrm{GeV}$ & Max & Mean & $S_{2}$ & $S_{3}$ \\
\hline$\mu^{-} \bar{\nu}_{\mu} u \bar{d}$ & 161 & 79.98 & 79.77 & 1.08 & -0.31 \\
$\mu^{-} \bar{\nu}_{\mu} u \bar{d}$ & 175 & 80.23 & 80.22 & 1.12 & -0.02 \\
$\mu^{-} \bar{\nu}_{\mu} u \bar{d}$ & 190 & 80.24 & 80.25 & 1.12 & 0.02 \\
$e^{-} \bar{\nu}_{e} u \bar{d}$ & 161 & 79.97 & 79.76 & 1.08 & -0.32 \\
$e^{-\bar{\nu}_{e}} u \bar{d}$ & 175 & 80.22 & 80.22 & 1.12 & -0.02 \\
$e^{-} \bar{\nu}_{e} u \bar{d}$ & 190 & 80.24 & 80.25 & 1.12 & 0.01 \\
\hline
\end{tabular}

Table 1: Moments of the $M_{+}=M(u \bar{d})$ distribution.

In addition, for the semi-leptonic case we have considered the following distributions

$$
\frac{d \sigma}{d E_{\gamma}}, \quad \frac{d \sigma}{d E_{l}}, \quad \frac{d \sigma}{d \cos \theta_{l}}
$$

which are shown in Fig. 6, 7 and 8 respectively. Within our working scheme

$$
E_{\gamma}=\left(1-\frac{x_{+}+x_{-}}{2}\right) \sqrt{s}
$$

where in the c.m.s the $e^{ \pm}$momenta are $P_{ \pm}=x_{ \pm} p_{ \pm}$.

The $E_{l}$ distribution is again of some relevance in the $M_{W}$ measurement since it allows a precise determination of the lepton end-point $E_{ \pm}$,

$$
E_{ \pm} \approx \frac{1}{2} E_{b} \pm \frac{1}{2} \sqrt{E_{b}^{2}-M_{W}^{2}}
$$

where $E_{b}$ is the beam energy. 
Few words of comments are in order to explain the relevance of the angular distributions. For $l=\mu$ the cut imposed on the scattering angle is irrelevant from a theoretical point of view since we could as well compute the fully extrapolated cross section (CC10 diagrams). For $l=e$ however it is a completely different story. Here we are dealing with the so called CC20 diagrams with $t$-channel photons which induce an apparent singularity at zero scattering angle. This is of course can be cured by avoiding the approximation of massless fermions but actually there is more.

Any calculation for $e^{+} e^{-} \rightarrow 4$-fermions is only nominally a tree level approximation because of the presence of charged and neutral, unstable vector bosons and of their interaction with photons. Unstable particles require a special care and their propagators, in some channels, must necessarily include an imaginary part or in other words the corresponding $S$-matrix elements will show poles shifted into the complex plane. In any field-theoretical approach these imaginary parts are obtained by performing the proper Dyson resommation of the relative two-point functions, which at certain thresholds will develop the requested imaginary component. The correct recipe seems representable by a Dyson re-summation of fermionic self-energies where only the imaginary parts are actually included. As a result the vector boson propagators will be inserted into the corresponding tree level amplitudes with a $p^{2}$-dependent width. It has already been noticed by several authors [17]-18] that even this simple idea gives rise to a series of inconsistencies, which sometimes may give results completely inconsistent even from a numerical point. The fact is that the introduction of a width into the propagators will inevitably result, in some cases, into a breakdown of the relevant Ward identities of the theory with a consequent violation of some well understood cancellation mechanism. In the CC20 case the effect of spoiling a cancellation among diagrams results into a numerical catastrophe at very small scattering angles.

This simple fact is well illustrated by our calculation where, at various energies we have reported in Fig. $9 d \sigma / d \cos \theta_{e}$ for $10^{\circ} \geq \theta_{e} \geq 1^{\circ}$. The two upper curves (solid and dash) are computed in the usual tree level approximation. Already at $1^{o}$ we have a growth of two order of magnitude with respect to $10^{\circ}$, effect which would become dramatical had we extended our calculation to smaller angles. Actually we have performed this rather academic calculation in order to show that WPHACT and WTO agree well even in some unrealistic and numerically unstable situations. The solution of this apparent puzzle is by now well know and amounts to adopting the so-called Fermion-Loop scheme [18]. The two lower curves (dotted and chain-dot) include these contributions. From the figure can be easily seen however, that no appreciable difference between the approximate and correct computation is present if a reasonable cut, of about $5^{\circ}$ or greater, is applied.

Moreover we have shown in Fig. 10 the total cross section for the two semi-leptonic processes as a function of the cut on the $l^{-}$scattering angle, $\theta_{\text {cut }}$. Here one can appreciate the difference between $e$ and $\mu$ when $\theta_{\text {cut }}$ goes to zero.

\section{$4 \quad$ Fully hadronic channel $\bar{q} q \bar{q} q$}

The fully hadronic channel has a substantial branching ratio and the typical topology consists of four (or more) energetic jets in the final state. It has been repeatedly stated 
in the literature that in the threshold region the ratio background/signal $\ll 1 \%$ but, in this respect, our analysis represents an attempt to quantify such a statement. As already mentioned in the introduction, the background coming from 2 gluons and 2 quarks and from other processes which could simulate a 4 quarks final state have not been considered in our computations.

First of all it is important to give a correct definition of signal and moreover we need an operative procedure to construct invariant mass distributions. As for the total cross section we have adopted the following algorithm. Let us arbitrarily denote by $i=1, \ldots, 4$ the four final state quarks, then we will have 6 different invariant masses $M_{i j}, i<j=1, \ldots, 4$. We will compute a cross section $\sigma(s)$ by requiring that

- $M_{12}$ and $M_{34}$. and/or. $M_{13}$ and $M_{24}$. and/or. $M_{14}$ and $M_{23}$ are within $10 \mathrm{GeV}$ away from $M_{W}$ while the remaining invariant masses are above $5 \mathrm{GeV}$.

In order to define an invariant mass distribution we adopt the following algorithm

- For each process we construct

$$
\frac{d \sigma}{d M_{i}}, \quad i=1, \ldots, 3
$$

where

1. $M_{1}=M_{12}+M_{34},\left|M_{12(34)}-M_{W}\right| \leq 10 \mathrm{GeV}$

2. $M_{2}=M_{13}+M_{24},\left|M_{13(24)}-M_{W}\right| \leq 10 \mathrm{GeV}$

3. $M_{3}=M_{14}+M_{23},\left|M_{14(23)}-M_{W}\right| \leq 10 \mathrm{GeV}$,

- those distributions which correspond to

1. $M(d \bar{u})+M(c \bar{s})$ in $\mathrm{CC} 11$,

2. $M(d \bar{u})+M(u \bar{d})$ in $\operatorname{Mix} 43$

add up, with their multiplicity, to define the signal, while all the rest is by definition the background.

In this way we are able to make a quantitative statement on the effect of $\mathrm{NC}$ processes on $W W$ distributions. In the end our procedure amounts to compute three distributions for 7 processes, in order to fully account for the irreducible background to $W W \rightarrow \bar{q} q \bar{q} q$. The main conclusion of our study is that the $\mathrm{NC}$ background, $u \bar{u} c \bar{c}$ etc, is completely negligible whenever we apply something of the order of a $\pm 10 \mathrm{GeV}$ cut around the $W$ mass. The only small but not negligible background is coming from non-leading contributions of the $\mathrm{CC}$ and Mix families. Moreover the leading contribution of the CC family is completely dominated by the double-resonant diagrams, the so-called CC03 approximation, at least for the type of cuts that we have selected.

Since the effect of the NC processes is marginal whenever a $\pm 10 \mathrm{GeV}$ cut is applied we can concentrate for a while on the signal, i.e. on the two processes $e^{+} e^{-} \rightarrow d \bar{u} c \bar{s}, d \bar{u} u \bar{d}$. 
From a pure theoretical point of view in this processes it is possible to identify $M_{ \pm}$as $M_{c \bar{s}}$ or $M_{u \bar{d}}$. In order to understand the role of our cuts and the effects of the incorrectly found jet-jet combinations we have also compared the total cross sections computed with two different algorithms. For instance in $e^{+} e^{-} \rightarrow d \bar{u} u \bar{d}$ we used

A1 $M_{W}-10 \mathrm{GeV} \leq M(d \bar{u}), M(u \bar{d}) \leq M_{W}+10 \mathrm{GeV}$, while $M(d \bar{d}), M(u \bar{u}), M(d u)$ and $M(\bar{u} \bar{d}) \geq 5 \mathrm{GeV}$.

A2 $M_{d \bar{u}}$ and $M_{u \bar{d}}$. and/or. $M_{d \bar{d}}$ and $M_{u \bar{u}}$. and/or. $M_{d u}$ and $M_{\bar{u} \bar{d}}$ are within $10 \mathrm{GeV}$ of $M_{W}$ while the remaining invariant masses are above $5 \mathrm{GeV}$.

Differences are ranging from $1.9 \%$ at $\sqrt{s}=160 \mathrm{GeV}$ to $1 \%$ at $\sqrt{s}=175 \mathrm{GeV}$ to $1.2 \%$ at $\sqrt{s}=190 \mathrm{GeV}$. The total cross section with the A2 algorithm is again reported in Fig. 2. The dash-dotted line refers to the signal, i.e to the CC11 and Mix43 processes while the solid line gives the total, therefore including all the NC32+NC64 background which becomes appreciable from energies slightly below the $Z Z$ threshold.

As far as the multiplicity of all channels is concerned, it is important to realize that if one takes into account the mixing induced by the CKM matrix then several other final states come into play, but the net result is just the same as not considering CKM, so one can really avoid considering these different processes for the present analysis. To show this, let us start from the naive case in which there is no CKM mixing matrix. Just counting the number of different processes with the same cross sections, one deduces the following set of weights:

$$
\begin{array}{lll}
W(u \bar{d} s \bar{c})=2, & W(u \bar{u} d \bar{d})=2, \\
W(u \bar{u} c \bar{c})=1, & W(u \bar{u} s \bar{s})=4, \\
W(d \bar{d} s \bar{s})=3, & W(u \bar{u} u \bar{u})=2, \\
W(d \bar{d} d \bar{d})=3, &
\end{array}
$$

This result is not affected by taking into account CKM mixing matrix. In fact for $\mathrm{CC} 11$, instead of considering only $u \bar{d} s \bar{c}$, one has now to sum over

$$
\begin{array}{lll}
u \bar{d} s \bar{c} \quad(d \bar{c}, b \bar{c}), & \left(V_{c d}\right)^{2}+\left(V_{c s}\right)^{2}+\left(V_{c b}\right)^{2}=1, \\
u \bar{s} s \bar{c} \quad(d \bar{c}, b \bar{c}), & \left(V_{c d}\right)^{2}+\left(V_{c s}\right)^{2}+\left(V_{c b}\right)^{2}=1, \\
u \bar{b} s \bar{c} \quad(d \bar{c}, b \bar{c}), & \left(V_{c d}\right)^{2}+\left(V_{c s}\right)^{2}+\left(V_{c b}\right)^{2}=1 .
\end{array}
$$

Since $\left(V_{u d}\right)^{2}+\left(V_{u s}\right)^{2}+\left(V_{u b}\right)^{2}=1$ we conclude that the sum of all these processes gives the same cross section as $u \bar{d} s \bar{c}$. For mixed processes, instead of the amplitudes for $u \bar{d} d \bar{u}$, $u \bar{s} s \bar{u}, u \bar{b} b \bar{u}$, one has now to evaluate

$$
\begin{aligned}
& u \bar{d} d \bar{u} \text { Amplitude }=N C+V_{u d}^{2} C C \\
& u \bar{d} s \bar{u} \text { Amplitude }=V_{u d} V_{u s} C C,
\end{aligned}
$$




$$
\begin{array}{ll}
u \bar{d} b \bar{u} \text { Amplitude }=V_{u d} V_{u b} C C, \\
u \bar{s} d \bar{u} \text { Amplitude }=V_{u d} V_{u s} C C, \\
u \bar{s} s \bar{u} \text { Amplitude }=N C+V_{u s}^{2} C C, \\
u \bar{s} b \bar{u} \text { Amplitude }=V_{u s} V_{u b} C C, \\
u \bar{b} d \bar{u} \text { Amplitude }=V_{u d} V_{u b} C C, \\
u \bar{b} s \bar{u} \text { Amplitude }=V_{u s} V_{u b} C C, \\
u \bar{b} b \bar{u} \text { Amplitude }=N C+V_{u b}^{2} C C,
\end{array}
$$

The sum of the first,second and third 3 gives for the cross sections

$$
\begin{aligned}
& N C^{2}+2 V_{u d}^{2} N C \times C C+V_{u d}^{2} C C^{2}, \\
& N C^{2}+2 V_{u s}^{2} N C \times C C+V_{u s}^{2} C C^{2}, \\
& N C^{2}+2 V_{u b}^{2} N C \times C C+V_{u b}^{2} C C^{2} .
\end{aligned}
$$

The total is $3 N C^{2}+2 N C \times C C+C C^{2}$ which is as $u \bar{u} d \bar{d}(N C+C C), u \bar{u} s \bar{s}(N C), u \bar{u} b \bar{b}(N C)$. By changing $u \rightarrow c$ all processes have been considered and indeed the weights correspond to the naive ones, without CKM mixing matrix.

There is another rather important issue to be discussed, namely to what extent is the double-resonant approximation (the so called CC03 diagrams) a good approximation. This is entirely cut dependent and by comparing $4 \times \mathrm{CC} 03$ with $2 \times(\mathrm{CC} 11+\mathrm{Mix} 43)$ in the A1 algorithm we find very small differences, of the order of $0.1 \%$ from $\sqrt{s}=160 \mathrm{GeV}$ to $\sqrt{s}=205 \mathrm{GeV}$.

The CC03 approximation is an important issue which has been debated at length. What we claim here is twofold, on one end we have produced an explicit and complete calculation up to including all fully hadronic processes (a part from the irreducible background $\bar{q} q g g$ ) where the goodness of the approximation can be quantitatively tested. On the other end the goodness of the approximation depends on the chosen set of cuts and even what can be considered reliable for a study of the $W$ mass is not also necessarily reliable for the full content of the four-fermion physics. Moreover the value of the distributions at $M(2 j)+M\left(2 j^{\prime}\right)=M_{\max }$ clearly shows that the CC11 and Mix43 background is not completely negligible. One last comment concerns the role to be played by dedicated four-fermion codes. From the LEP 1 experience we know that one of the possible working options has been to de-convolute the experimental data and to use the result for fitting the parameters of the standard model. Of course to create a 4 f-fitter requires very high computational speed associated with reasonably high precision. Both WPHACT and WTO can deal with semi-leptonic and fully hadronic processes in an efficient way and, in particular, they are extremely fast in dealing with CC11-Mix43. Therefore they both could be interfaced with some fitting procedure resulting in a fast and accurate determination of the standard model parameters.

In Fig. 11-13 we have reported the distribution in the sum of two invariant masses according to the algorithm previously discussed and making a distinction between signal, CC11 + Mix43 background and NC background. The latter has been magnified by a factor of 50 while the CC11 + Mix43 one by a factor of 5 . Even if this method will 
probably not be used at $161 \mathrm{GeV}$, we report the curves at such energy in Fig. 11 for completeness.

Our algorithm is based on the general observation that at large $M_{W} / \sqrt{s}$ one has an excellent determination of $M(2 j)+M\left(2 j^{\prime}\right)$ while $M(2 j)-M\left(2 j^{\prime}\right)$ is poorly determined. Therefore in the 4-jet channel we never ask which jet is reconstructed and we use all (three) possible combinations. The additional cut of $M_{W} \pm 10 \mathrm{GeV}$ simulates in a wide enough mass window the (almost) equal mass constraint.

This theoretical simulation of the experimental data handling tells us that the selected cuts are enough to make the NC background safely neglected. The remaining effect can be understood from table 2 where we report some of the moments for the $M(2 j)+M\left(2 j^{\prime}\right)$ distribution in $e^{+} e^{-} \rightarrow 2 j+2 j^{\prime}$.

\begin{tabular}{|c|c|c|c|c|c|}
\hline Final state & $\sqrt{s} \mathrm{GeV}$ & Max & Mean & $S_{2}$ & $S_{3}$ \\
\hline total & 161 & 159.46 & 157.96 & 2.82 & -2.01 \\
total & 175 & 160.40 & 160.34 & 2.95 & -0.44 \\
total & 190 & 160.42 & 160.54 & 3.03 & 0.25 \\
signal & 161 & 159.48 & 158.27 & 2.29 & -1.66 \\
signal & 175 & 160.40 & 160.36 & 2.48 & -0.26 \\
signal & 190 & 160.42 & 160.51 & 2.55 & 0.03 \\
\hline
\end{tabular}

Table 2: Moments of the $M(2 j)+M\left(2 j^{\prime}\right)$ distribution.

The previous results obtain for $M_{W}=80.26 \mathrm{GeV}$. Within the minimal standard model and within our renormalization scheme we obtain

$$
\Gamma_{W}=2.0902 \mathrm{GeV}, \quad\left(\alpha_{s}=0.1255\right) .
$$

Therefore one easily obtains that in the double-resonant (CC03) approximation and without initial state QED radiation the maximum of such distributions should be at

$$
M_{\max }=2 \frac{M_{W}^{2}}{\left(M_{W}^{2}+\Gamma_{W}^{2}\right)^{1 / 2}}=160.47 \mathrm{GeV} .
$$

From table 2 we can easily reconstruct the effect of the background and of initial state radiation and kinematical cuts.

In this paper we have made no attempt to give a detailed description of the theoretical uncertainties associated with four fermion production. However on some specific issue we 
can point out possible sources of discrepancy which indeed reflect a theoretical uncertainty. Once we agree on applying the NQCD prescription we still face two basic options for its implementation in fully hadronic channels. Given four quarks in the final state NQCD could amount to multiply by $\left(1+\alpha_{s} / \pi\right)^{2}$ but one could also decide to linearize. Since $\left(\alpha_{s} / \pi\right)^{2}$ is of the order of $1 \div 2$ permill the difference will show up (cfr. Fig. 1) in any comparison which is aimed to a $0.1 \div 0.2$ permill, as the one that we have constantly performed.

Actually there is more in the application of NQCD to fully hadronic processes. The typical pattern that we have to analyze is the following. First of all NQCD amounts to neglecting kinematical cuts and to allow for QCD radiation from external quark lines only. Thus the main approximation concerns neglecting radiation from internal lines. Even in this approximation we have quarks which are connected to $W$ and $Z$ bosons, to photons and to gluons. What to choose for the corresponding scale $\mu$ at which $\alpha_{s}$ is evaluated? Basically we have made a distinction among three possibilities, all equally plausible and naive.

1. For this particular class of processes we fix $\mu$ to be $M_{W}$ for CC processes and $M_{Z}$ for NC processes.

2. Still we may choose to apply NQCD everywhere or only in double-resonating approximation, which means that only $W W$ or $Z Z$ channels are corrected.

3. We adopt a more ambitious program. Each external $\bar{q} q$ pair is characterized by its invariant mass, no matter where it is coming from, thus we include NQCD with a correction factor proportional to $\alpha_{s}\left(m_{\bar{q} q}\right)$.

For the range of energies and of kinematical cuts implied by the present analysis it turns out that the three previous options lead to negligibly small differences.

\section{Conclusions}

Given the intrinsic relevance of having the most reliable predictions for $W$ physics at LEP 2 energies and strongly motivated by the success of several numerical comparisons at the recent LEP 2 working groups we have used two four fermion dedicated FORTRAN codes, WPHACT and WTO, to perform a careful a detailed analysis of the distributions in those four fermion processes which are relevant at LEP 2 for the measurement of the $W$ mass and for the predictions of the standard model concerning the production of two $W$ bosons.

The codes use completely different techniques for evaluating the matrix elements, for the phase space integration and for producing distributions. The perfect (technical) agreement obtained and the smallness of the statistical errors enforces the reliability of the results. A word of caution should however be spent to recall that these computations are always affected by a theoretical uncertainty which has been estimated [2] to be around a few per mille and which has different roots connected to the choice of the input parameters $\left(\alpha_{Q E D}\right.$ versus $\left.G_{\mu}\right)$, of the renormalization scheme, of the treatment of initial and final state 
QED radiation, of the application of approximate final state QCD correction factors. Moreover the computations are always at the parton level: no hadronization has been introduced and all cuts refer to the partons.

It is our opinion that, given the uncertainties connected to the hadronization processes, it is necessary to have the most accurate predictions at the parton level, in order to disentangle the perturbative regime from the non perturbative one. When the hadronization programs will be fully tuned also at LEP 2 and all the problems connected to either colour reconnection or to Bose-Einstein effect will be completely under control then it will probably be possible to de-convolute the data from the hadronization and to compare them with dedicated parton level predictions such as those that we have given in our analysis and that could eventually form a basis for some fitting procedure similar the the one which has become so popular at LEP 1. From this point of view we have shown that all the requirements of computational speed and of technical precision have been fulfilled.

The results of our study are summarized by the distributions themselves. They confirm and complete some of the results reported in ref. [19] by using a different and complementary approach. Moreover, we have proposed some particular algorithm for the fully hadronic channels in order to give an unambiguous definition of the signal, of the irreducible four-quarks background and of the procedure to construct invariant mass distributions even in the absence of flavor reconstruction.

We have shown that with appropriate kinematical cuts one can actually dispose of the Neutral Current background when the problem of determining the $W$ boson mass is addressed. On the other hand, the background from the complete Charge Current and Mixed processes is not completely negligible. We have also considered all processes induced by the CKM mixing and concluded that introducing them is superfluous for this kind of analyses.

For processes which have an electron in the final state, the so called CC20 diagrams, we have carefully addressed the questions related to numerical instability and to gauge restoration, therefore giving one of the few practical and reliable implementations for these processes at small scattering angle of the electron also confirming that an angular cut around $10^{\circ}$ down to approximately $5^{\circ}$ will suffice in guaranteeing reliable predictions even without having to use a gauge restoring scheme.

\section{References}

[1] Physics at LEP2, G. Altarelli T. Sjostrand and F. Zwirner eds., CERN 96-01

[2] Report on Event Generators for WW Physics in ref. [1]

[3] F.A. Berends, P.H. Daverveldt, R. Kleiss, Nucl.Phys. B253(1985)441;

D. Bardin, M. Bilenky, A. Olchevski and T. Riemann, Phys. Lett. B308(1993)403; E:[ibid.B357(1995)725];

T. Ishikawa, T. Kaneko, S. Kawabata, Y. Kurihara, Y. Shimizu, H. Tanaka, prep. KEK-92-210, Feb 1993;

F.A. Berends, R. Kleiss, R. Pittau, Nucl.Phys. B424(1994)308;

Nucl.Phys. B426(1994)344; Nucl.Phys. B, Proc. Suppl. 37B(1994)163; 
Y. Kurihara, D. Perret-Gallix and Y. Shimizu, Phys. Lett. B349(1995)367;

G. Montagna, O. Nicrosini, G. Passarino and F. Piccinini, Phys. Lett. B348(1995)178;

C. G. Papadopoulos, Phys. Lett. B352(1995)144;

D. Bardin, M. Bilenky, D. Lehner, A. Olchevski and T. Riemann,

Nucl. Phys. (Proc. Suppl.) 37B(1994)148;

D. Bardin and T. Riemann, Nucl. Phys. B462(1996)3;

F. Caravaglios, M. Moretti Oxford prep. OUTP-96-13-P, Apr 1996.

[4] E. Boos, M. Sachwitz, H.J. Schreiber, S. Shichanin, Z. Phys. C61(1994)675;

Z. Phys. C64(1994)391; Int. J. Mod. Phys. A10(1995)2067;

Z. Phys. C67(1995)613; prep. DESY-96-007, Jan 1996;

M. Dubinin, V. Edneral, Y. Kurihara, Y. Shimizu, Phys. Lett. B329(1994)379;

D. Bardin, A. Leike, T. Riemann, Phys. Lett. B344(1995)383;

Phys. Lett. B353(1995)513;

D. Apostolakis, P. Ditsas, S. Katsanevas, prep. CRETE-96-12, Mar 1996;

G. Montagna, O. Nicrosini, F.Piccinini, Phys. Lett. B348(1995)496;

[5] T. Sjöstrand, Comp. Phys. Commun. 82(1994)74;

G. J. van Oldenborgh, P. J. Franzini, A. Borrelli, Comp. Phys. Commun. 83(1994)14;

F.A. Berends, R. Pittau, R. Kleiss, Comp. Phys. Commun. 85(1995)437;

F. Caravaglios, M. Moretti, Phys. Lett. B358(1995)332;

M. Skrzypek, S. Jadach, W. Placzek, Z. Was, prep. CERN-TH-95-205, Jul 1995;

G. Montagna, O. Nicrosini, F. Piccinini, Comp. Phys. Commun. 90(1995)141;

H. Anlauf, P. Manakos, T. Ohl, H. D. Dahmen, prep. IKDA-96-15, May 1996;

J. Fujimoto, T. Ishikawa, T. Kaneko, K. Kato, S. Kawabata, Y. Kurihara, T. Munehisa, D. Perret-Gallix, Y. Shimizu, H. Tanaka, prep. KEK-CP-046, May 1996 ;

[6] G. Passarino, hep-ph/9602302(1996), to appear in Comp. Phys. Comm;

E. Accomando and A. Ballestrero, WPHACT 1.0 - A program for $W W$, Higgs and 4 fermion physics at $e^{+} e^{-}$colliders, Torino prep. DFTT 16/96 in preparation.

[7] Reports on Event Generators for Discovery Physics in ref. [1]

[8] Report on Standard Model Processes in ref. [1]

[9] Report on WW Cross-sections and distributions in ref. [1]

[10] Report on QCD Even Generators in ref. [1]

[11] A. Ballestrero, E. Maina, S. Moretti, Nucl.Phys. B415(1994)265;

R. Pittau, Phys. Lett. B335(1994)490.

[12] A. Ballestrero, in preparation.

[13] A. Ballestrero and E. Maina, Phys. Lett. B350(1995)225. 
[14] G.P. Lepage, Jour. Comp. Phys. 27(1978)192.

[15] G. Passarino, Nucl. Phys. B237(1984)249.

[16] Reports of the Working Group on Precision Calculations for the Z Resonance, D. Bardin, W. Hollik, G. Passarino eds., CERN 95-03

[17] M. Veltman, Physica 29(1963)186;

F. A. Berends and G. B. West, Phys. Rev. D1(1970)122;

R. G. Stuart, Phys. Lett. B262(1991)113;

A. Aeppli, C. J. van Oldenborgh and D. Wyler, Nuc. Phys. B248(1994)126;

U. Baur and D. Zeppenfeld, Phys. Rev. Lett. 75(1995)1002;

[18] E. N. Argyres et al., Phys Lett. B358(1995)339.

[19] Report on Determination of the mass of the $W$ boson in ref. [1] 

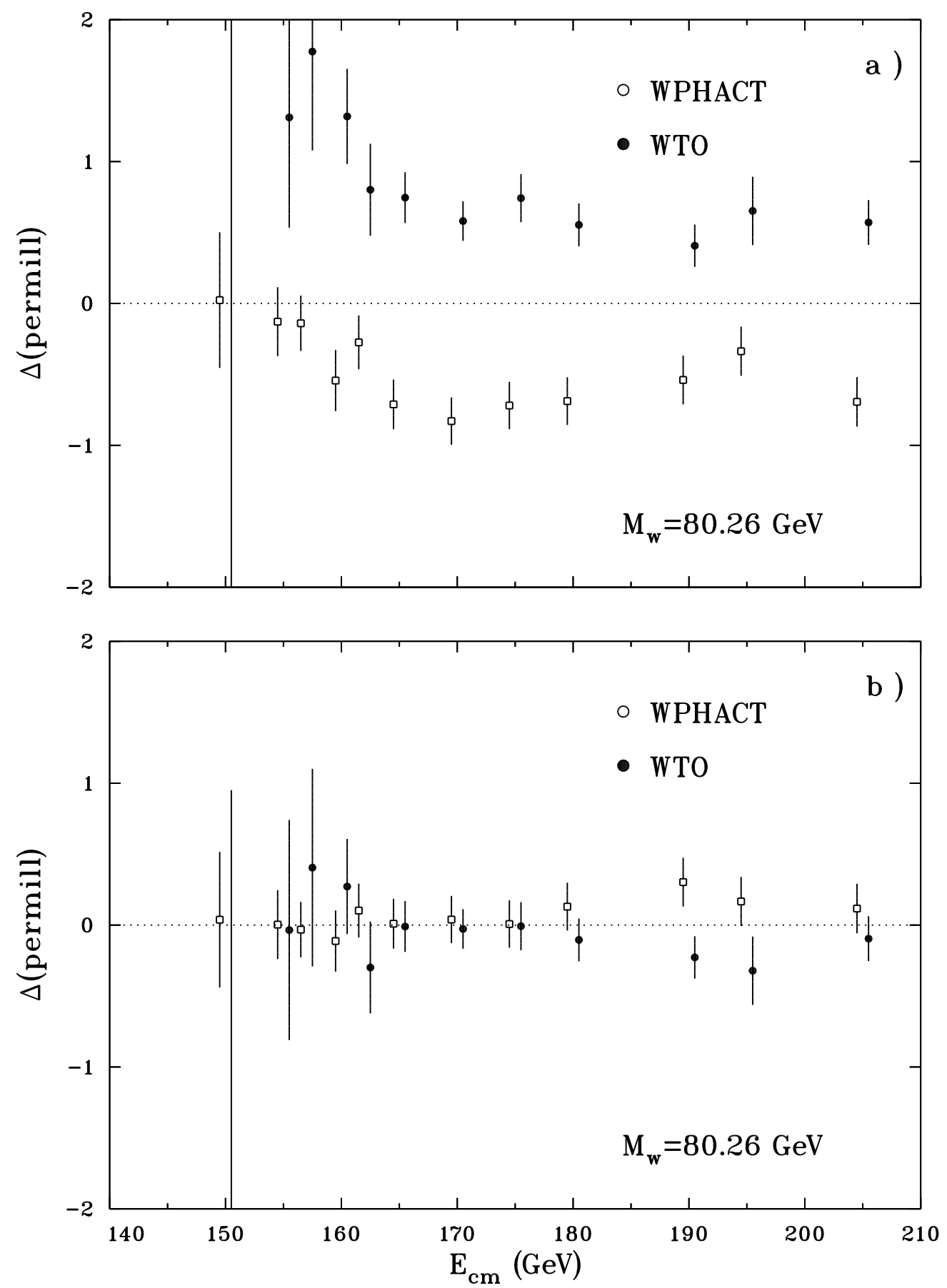

Fig.1- Deviations of the WPHACT and WTO results, from their weighted average versus $\sqrt{s}$, for $u \bar{d} s \bar{c}$ cross-section. The upper plot corresponds to the comparison with NQCD implementations differing of order $\left(\alpha_{s} / \pi\right)^{2}$, the lower to the same NQCD implementations. 


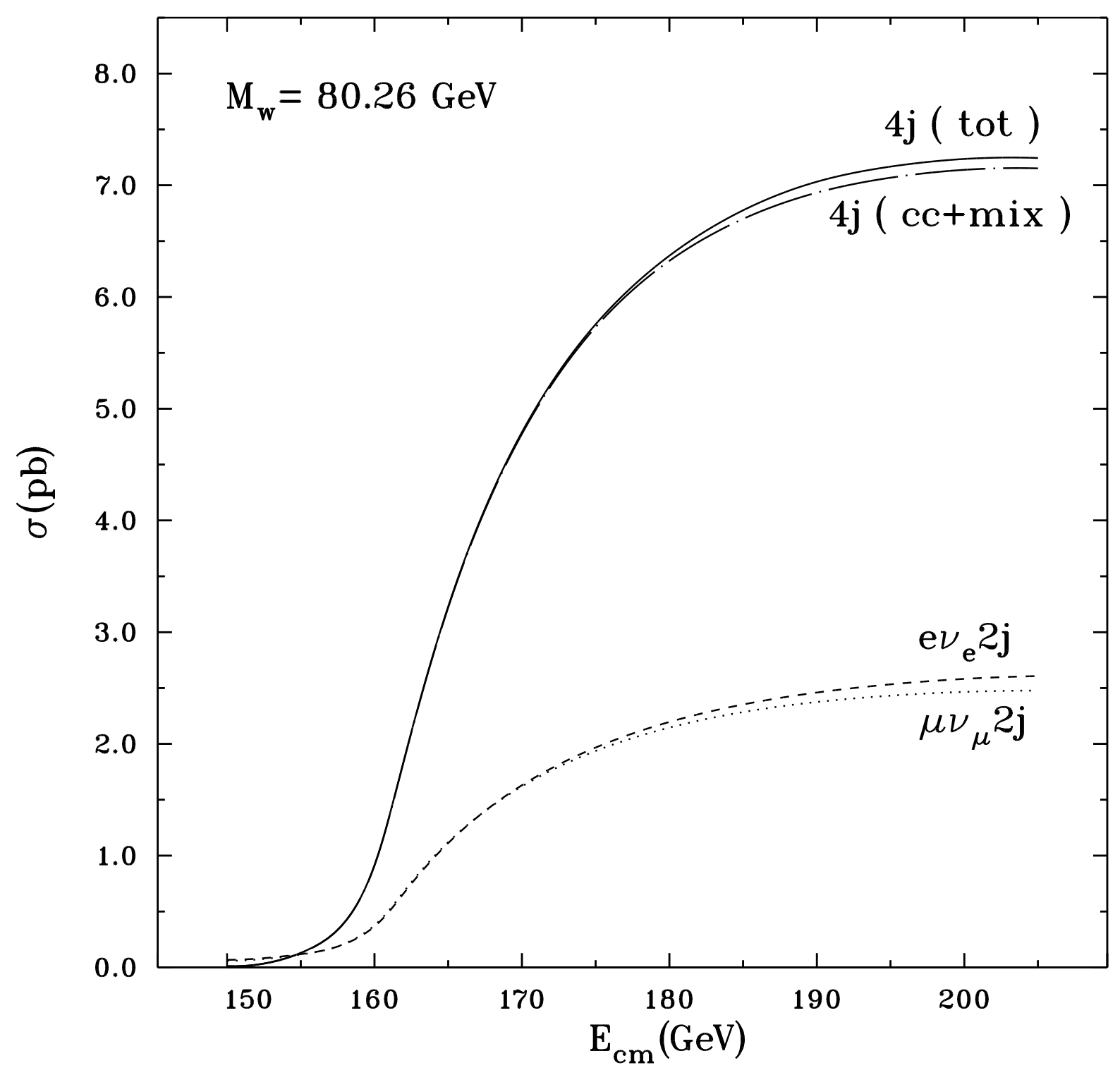

Fig.2- Total cross-section versus $\sqrt{s}$, for the semi-leptonic channels $q \bar{q} e \nu_{e}$ (dashed line) and $q \bar{q} \mu \nu_{\mu}$ (dotted line) with canonical cuts, and for the fully hadronic $q_{1} \bar{q}_{2} q_{3} \bar{q}_{4}$ signal (chaindot line) and signal $+\mathrm{NC}$ background (solid line) with the constraints: a) $E_{i}>3$ $\mathrm{GeV}, \mathrm{i}=1 . .4$, b) $M_{12}$ and $M_{34}$, and/or, $M_{13}$ and $M_{24}$, and/or, $M_{14}$ and $M_{23}$ within 10 $\mathrm{GeV}$ away from $M_{W}$, c) the remaining invariant masses above $5 \mathrm{GeV}$. 


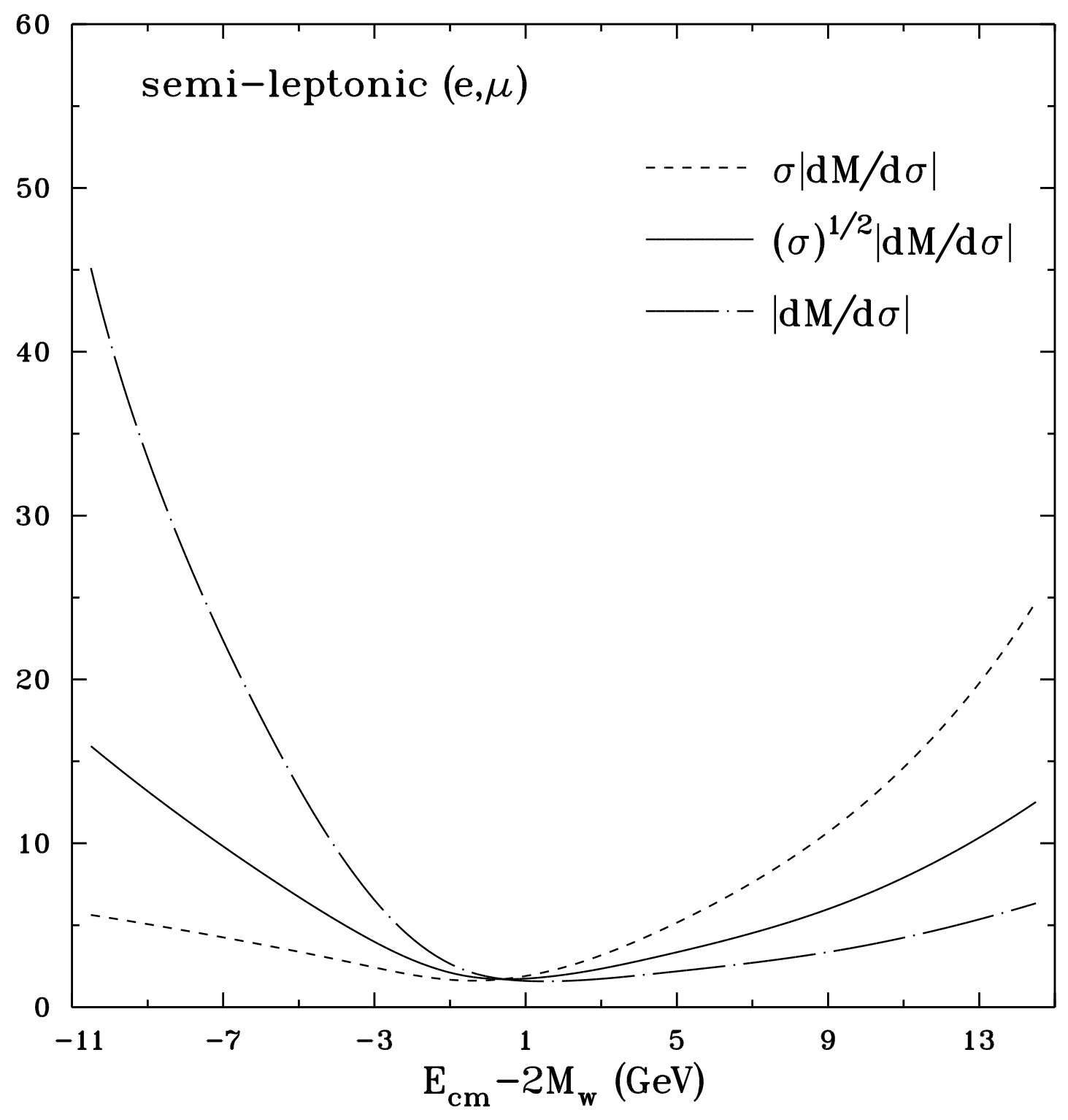

Fig.3- Statistical and systematic sensitivity factors to the W mass in the semi-leptonic channel as a function of $\sqrt{s}-2 M_{W}$, for $M_{W}=80.26 \mathrm{GeV}$. The connection of the three curves with the threshold measurement of $M_{W}$ is discussed in the text. 


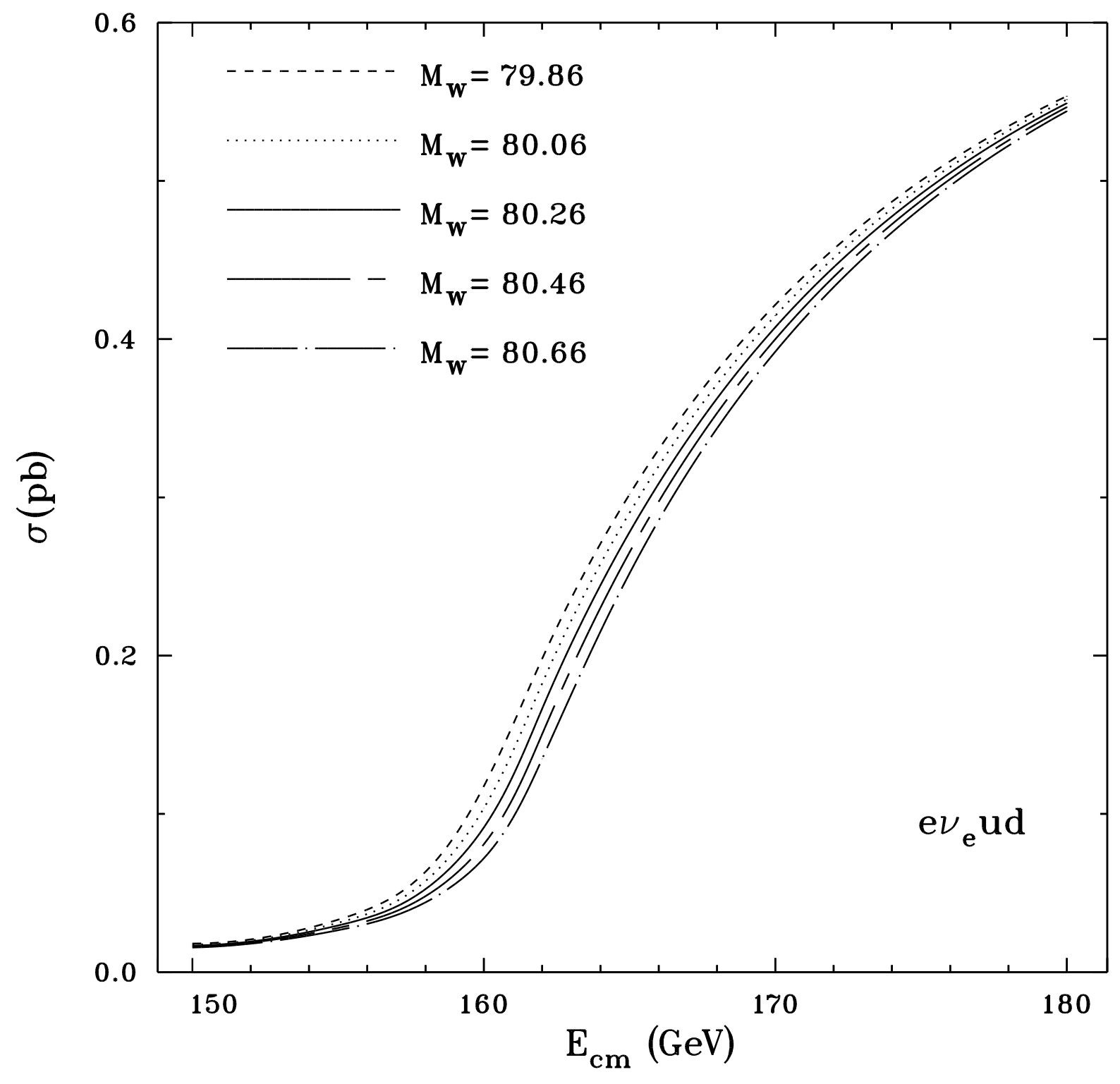

Fig.4- Total cross-section for $e^{-} \bar{\nu}_{e} u \bar{d}$ process, with canonical cuts, versus $\sqrt{s}$, from $150 \mathrm{GeV}$ to $205 \mathrm{GeV}$, for different input parameter $M_{W}$ values. 


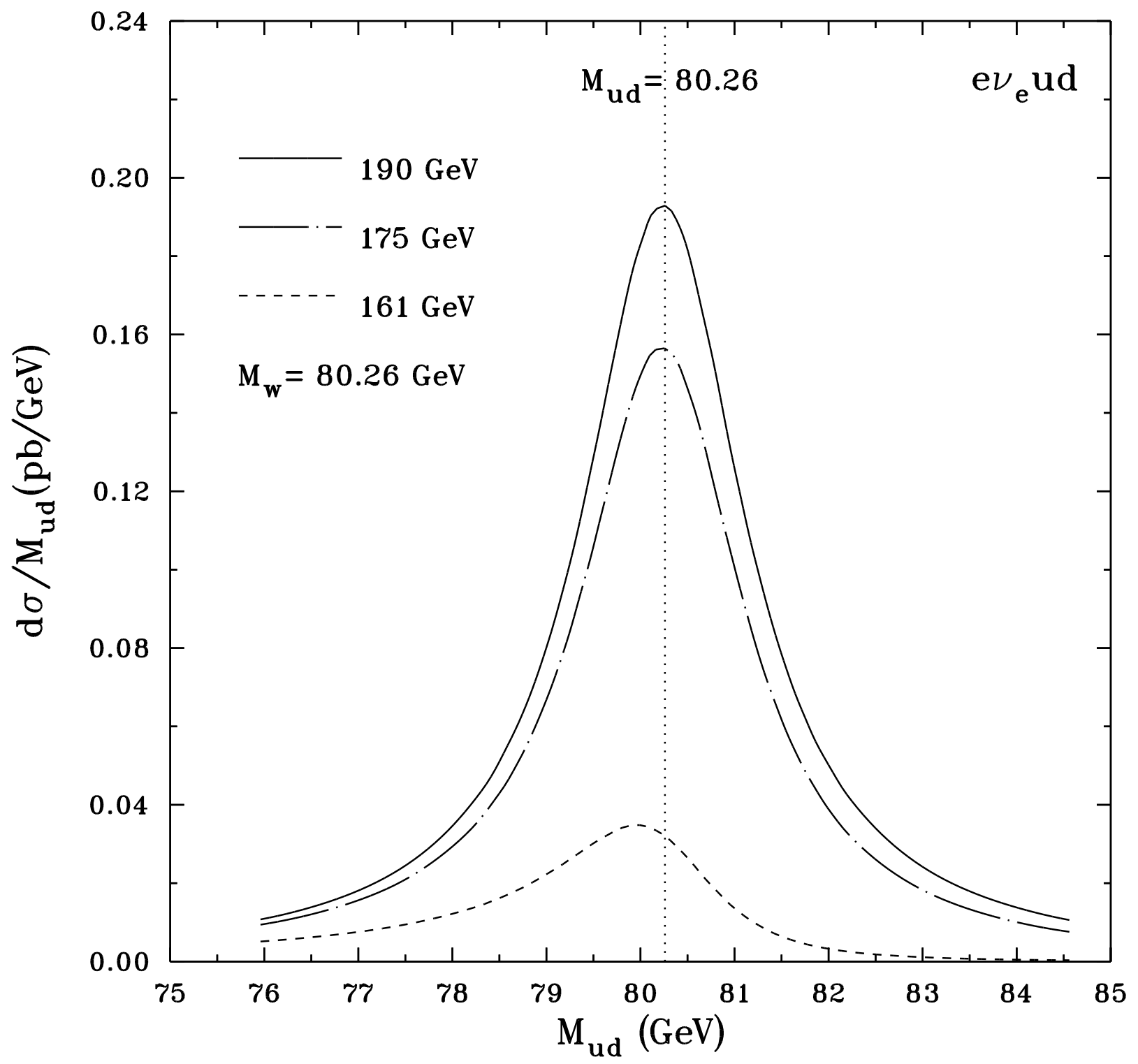

Fig.5- Invariant mass distribution of $u \bar{d}$ in the $e^{-} \bar{\nu}_{e} u \bar{d}$ process, with canonical cuts, at $\sqrt{s}=161 \mathrm{GeV}$ (dashed line), $175 \mathrm{GeV}$ (chaindot line) and $190 \mathrm{GeV}$ (solid line). 


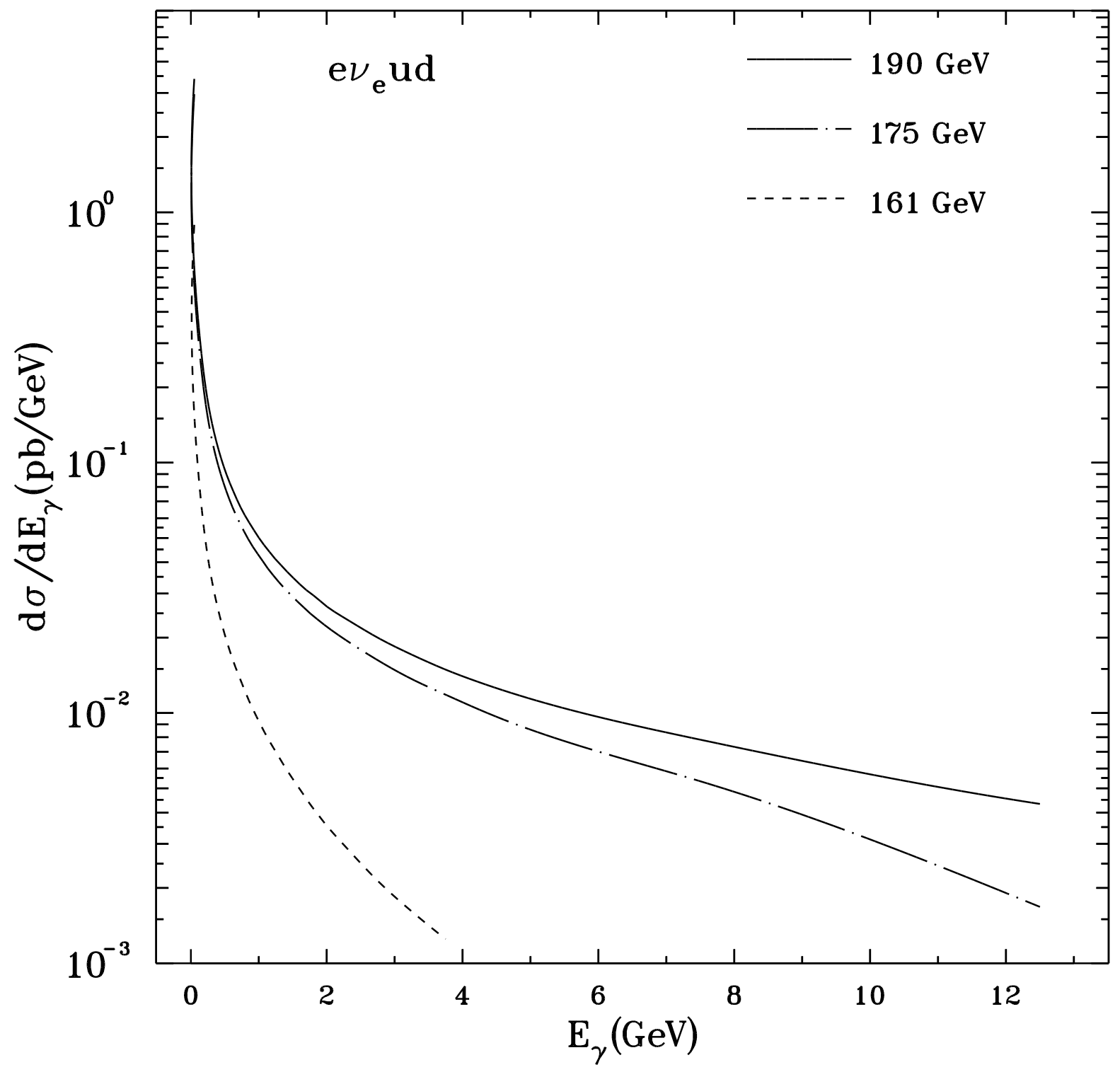

Fig.6- Energy spectrum of initial state photons in $e^{-} \bar{\nu}_{e} u \bar{d}$ process, with canonical cuts, at $\sqrt{s}=161 \mathrm{GeV}$ (dashed line), $175 \mathrm{GeV}$ (chaindot line) and $190 \mathrm{GeV}$ (solid line), for $M_{W}=80.26 \mathrm{GeV}$. 


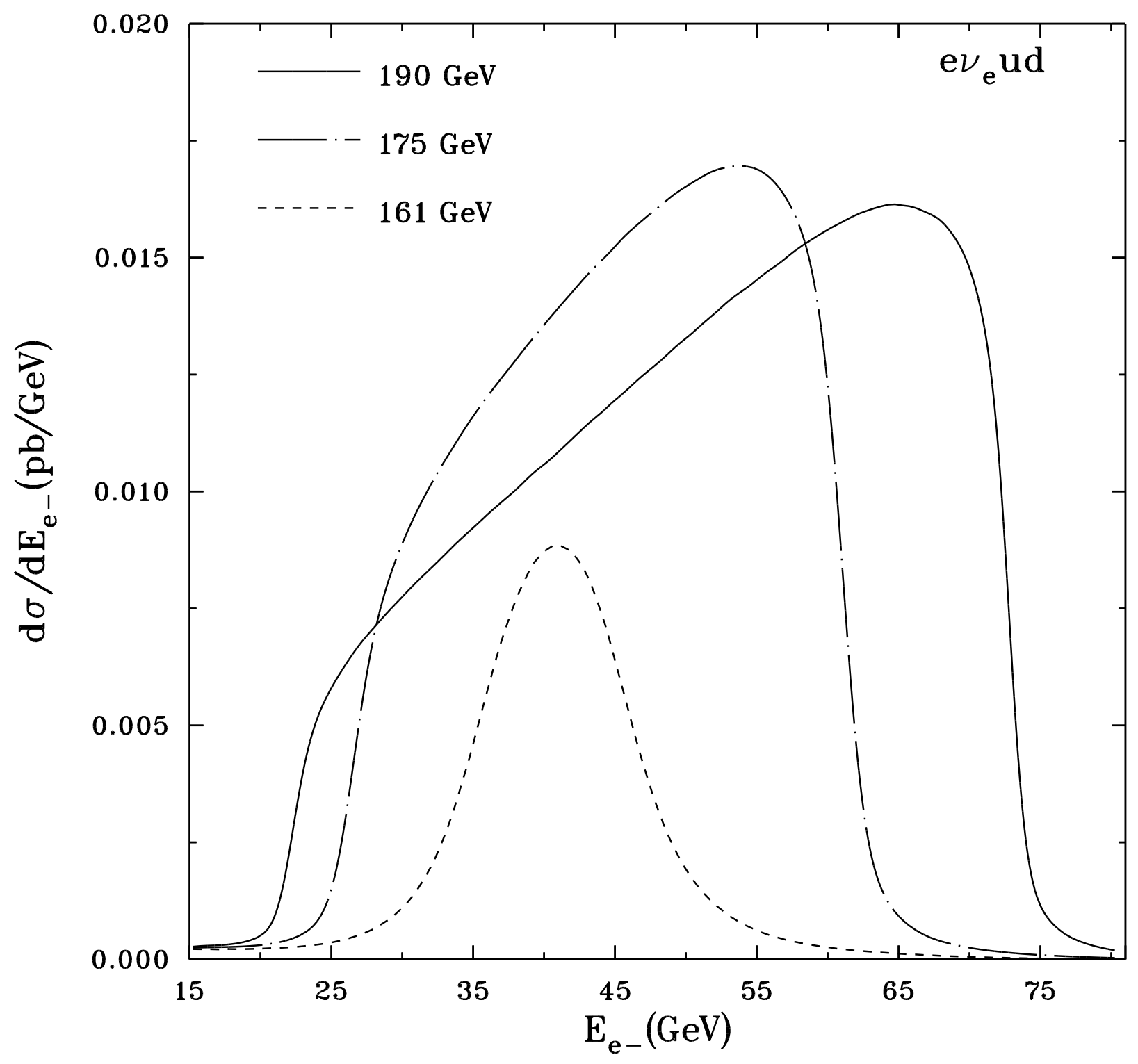

Fig.7- Distribution of the final state electron energy in the $e^{-} \bar{\nu}_{e} u \bar{d}$ process, with canonical cuts, at $\sqrt{s}=161 \mathrm{GeV}$ (dashed line), $175 \mathrm{GeV}$ (chaindot line) and $190 \mathrm{GeV}$ (solid line), for $M_{W}=80.26 \mathrm{GeV}$. 


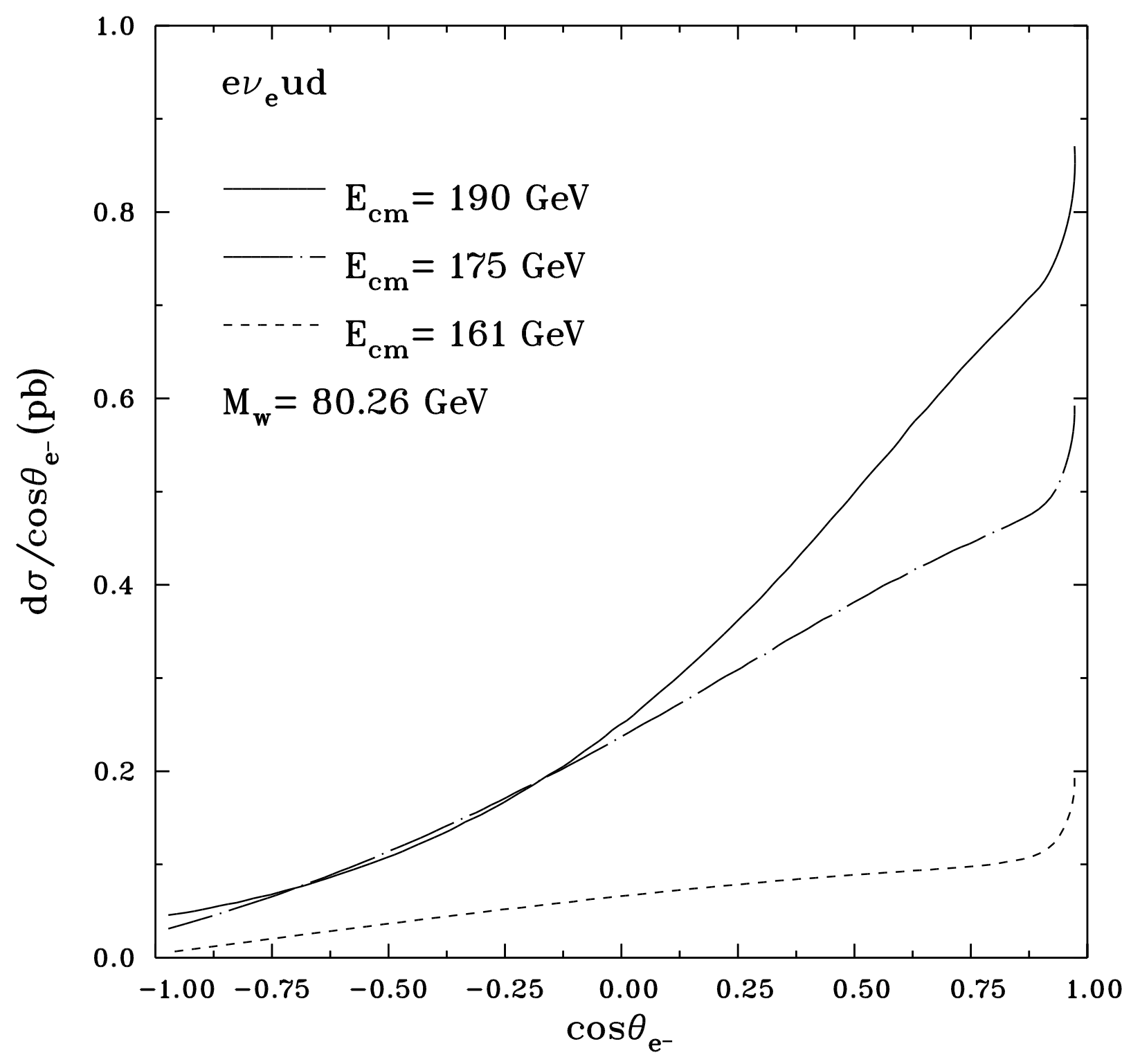

Fig.8- Angular distribution of the final state electron in the $e^{-} \bar{\nu}_{e} u \bar{d}$ process at $\sqrt{s}=161$ $\mathrm{GeV}$ (dashed line), $175 \mathrm{GeV}$ (chaindot line) and $190 \mathrm{GeV}$ (solid line). Canonical cuts are applied. 


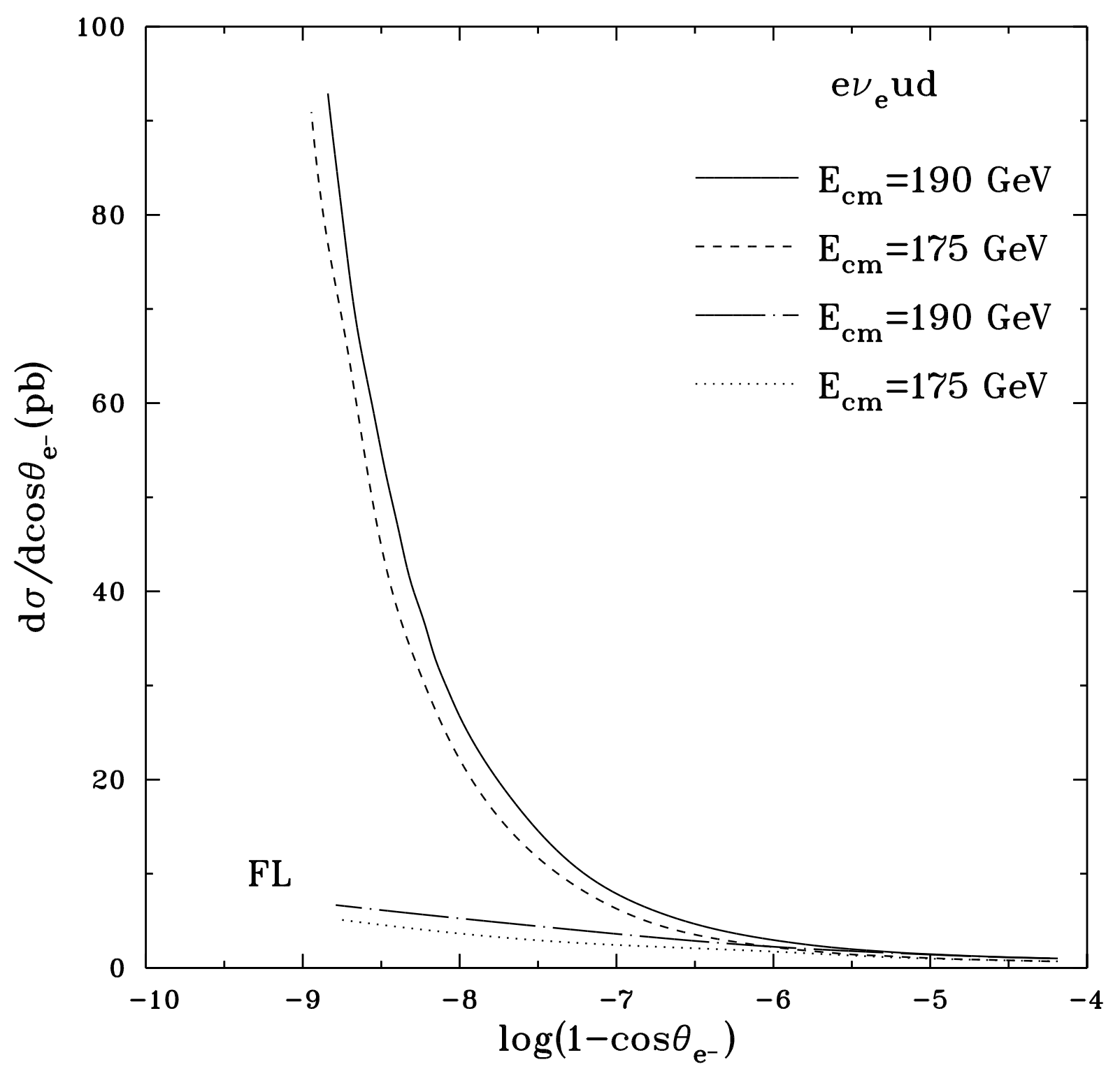

Fig.9- Angular distribution of the final state electron in $e^{-} \bar{\nu}_{e} u \bar{d}$, with $M_{W}=80.26$ $\mathrm{GeV}$, as a function of $\log \left(1-\cos \vartheta_{e^{-}}\right)$, for $10^{\circ} \geq \vartheta_{e} \geq 1^{o}$, at $\sqrt{s}=175 \mathrm{GeV}$ (dashed line) and $190 \mathrm{GeV}$ (solid line). The dotted and chaindot curves include the Fermion-Loop scheme at $\sqrt{s}=175 \mathrm{GeV}$ and $\sqrt{s}=190 \mathrm{GeV}$ respectively. Canonical cuts are applied. 


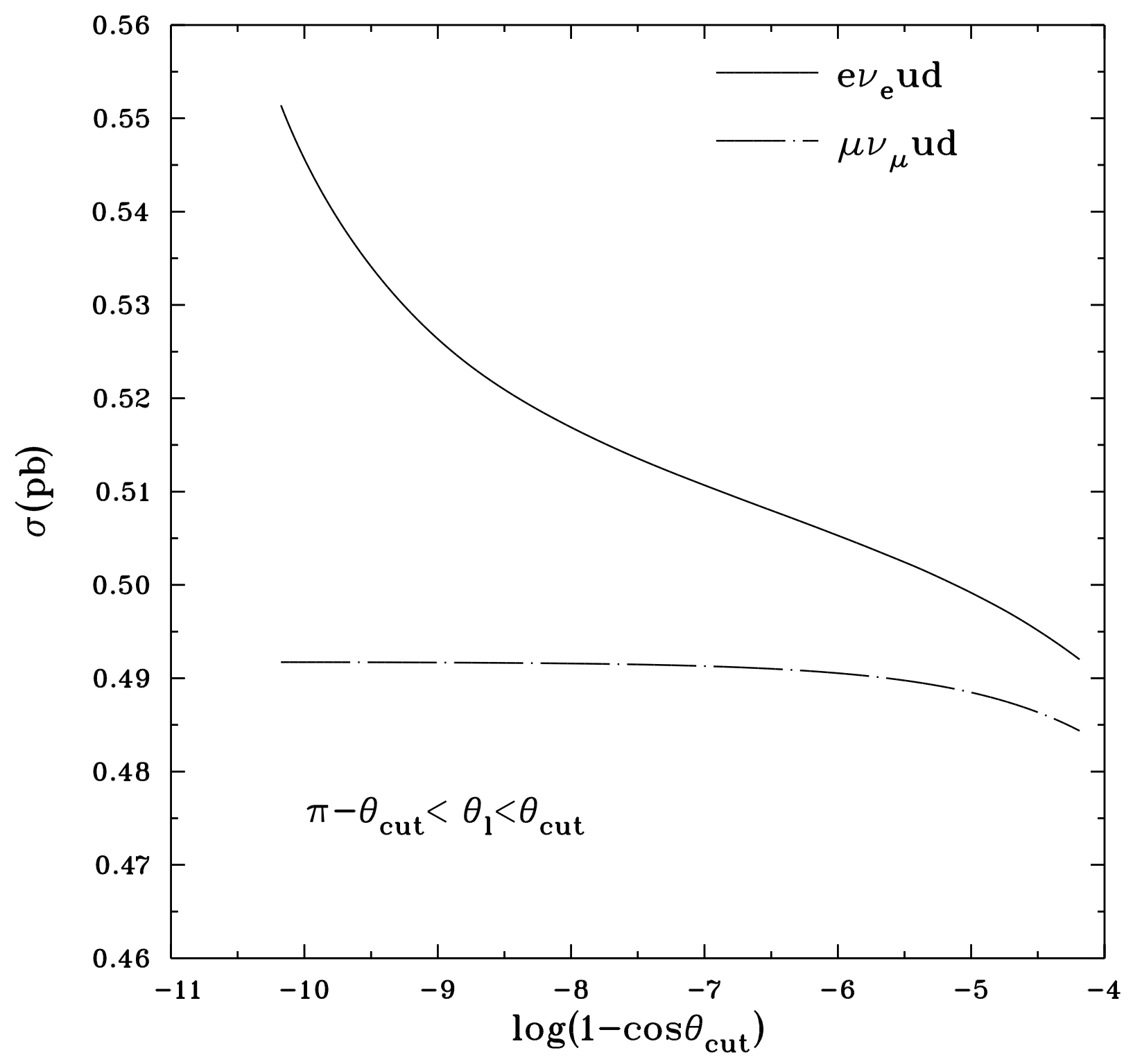

Fig.10- Total cross-section for the semi-leptonic processes $e^{-} \bar{\nu} e \bar{d}$ (solid line) and $\mu^{-} \bar{\nu}_{\mu} u \bar{d}$ (chaindot line) as a function of $\log \left(1-\cos \vartheta_{c u t}\right) . \quad \vartheta_{\text {cut }}$ the cut imposed on the charged lepton scattering angle. The remaining canonical cuts are preserved. $M_{W}=80.26$ $\mathrm{GeV}$. 


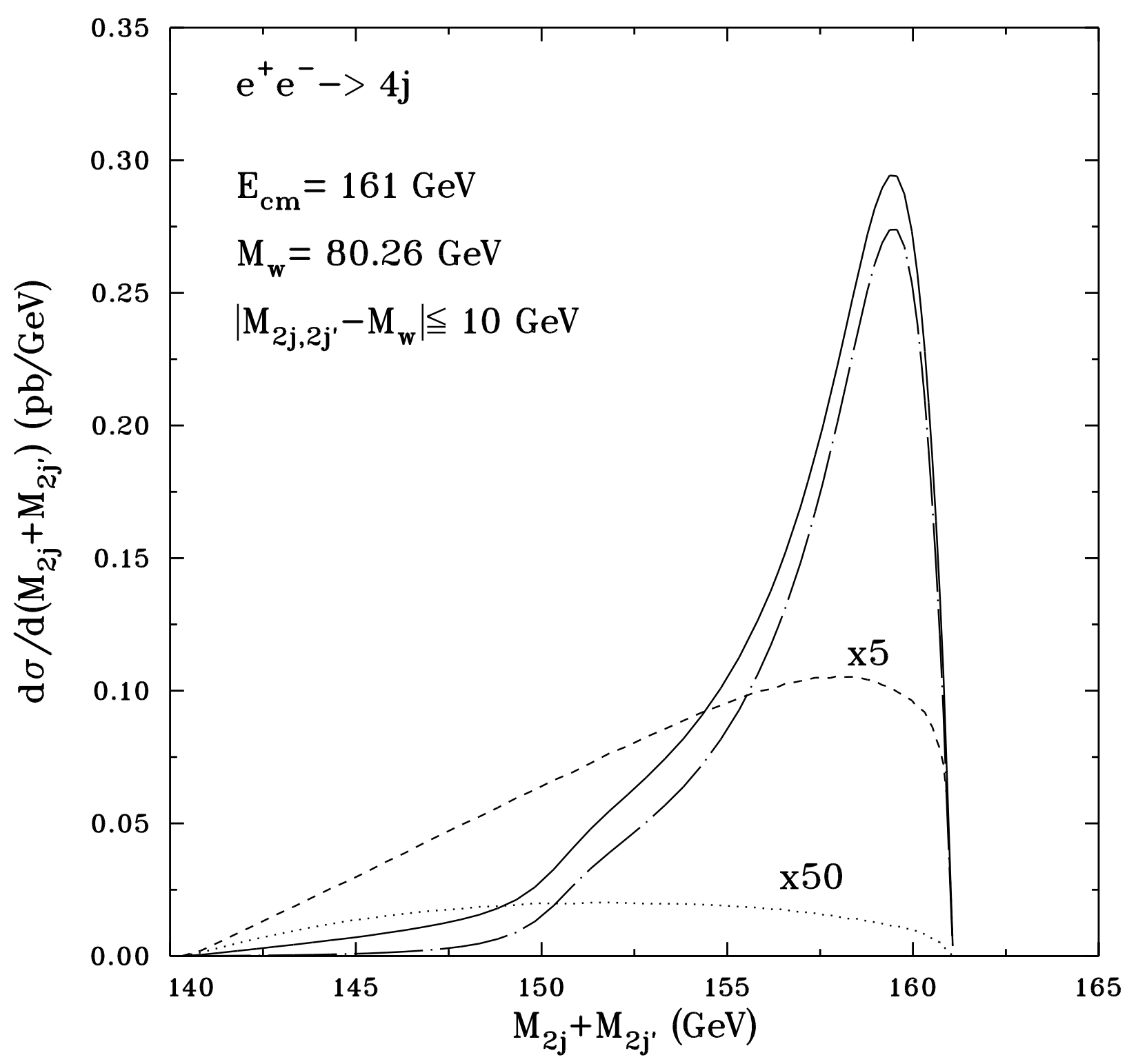

Fig.11- Distribution of the sum of two invariant masses in the fully hadronic channel at $\sqrt{s}=161 \mathrm{GeV}$. The chaindot curve corresponds to the two invariant masses from $W^{* \pm}$. The dashed one represents the background ( magnified by a factor of 5 ) from two nonresonant invariant masses in $\mathrm{CC} 11$ and Mix43 processes, counted with their molteplicity. The dotted curve corresponds to the NC background ( magnified by a factor 50 ). The solid to signal+total background. For each sum, the two invariant masses lie within 10 $\mathrm{GeV}$ from $M_{W}$ 


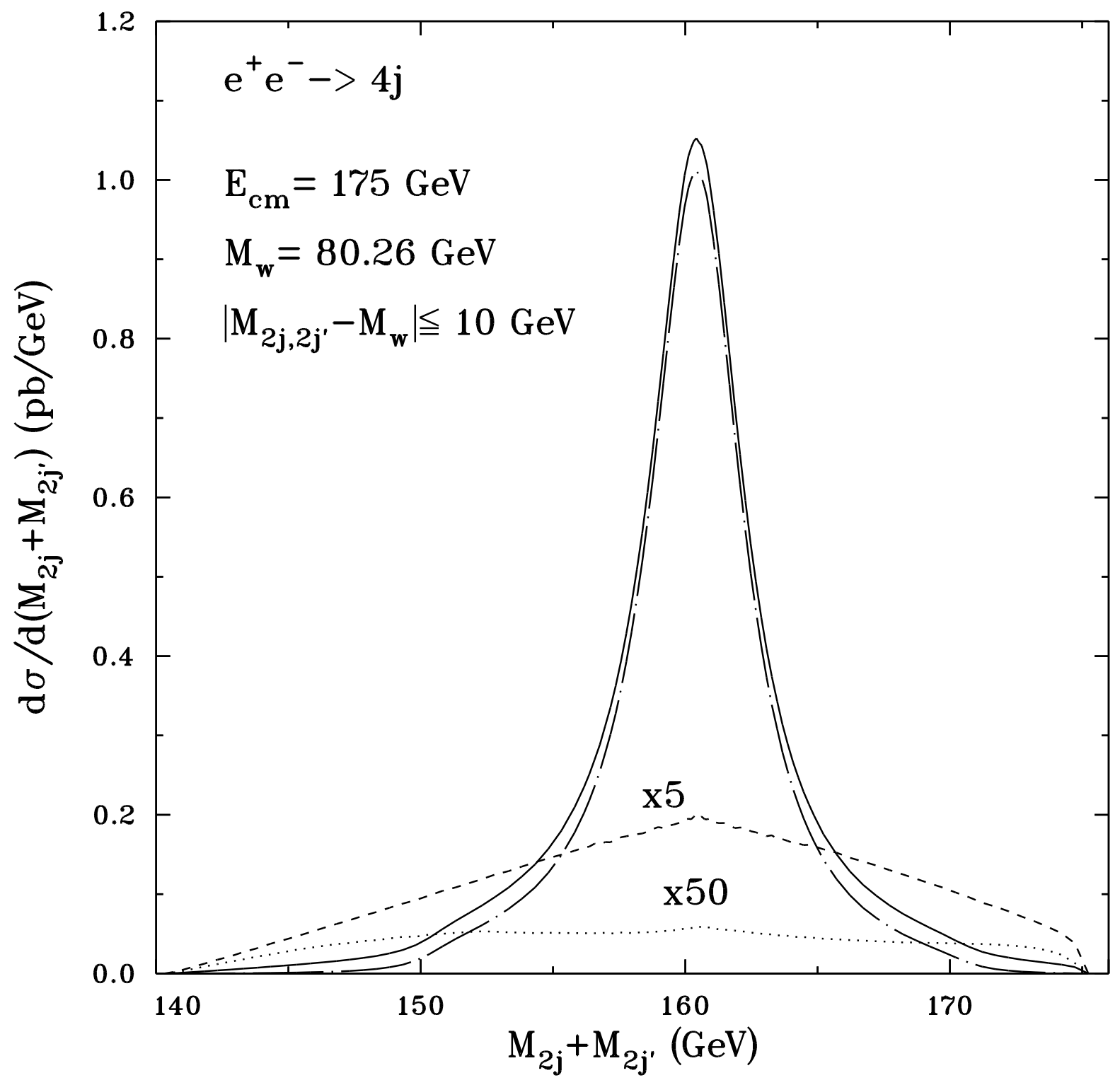

Fig.12- Same distribution as in Fig.11, with $\sqrt{s}=175 \mathrm{GeV}$. 


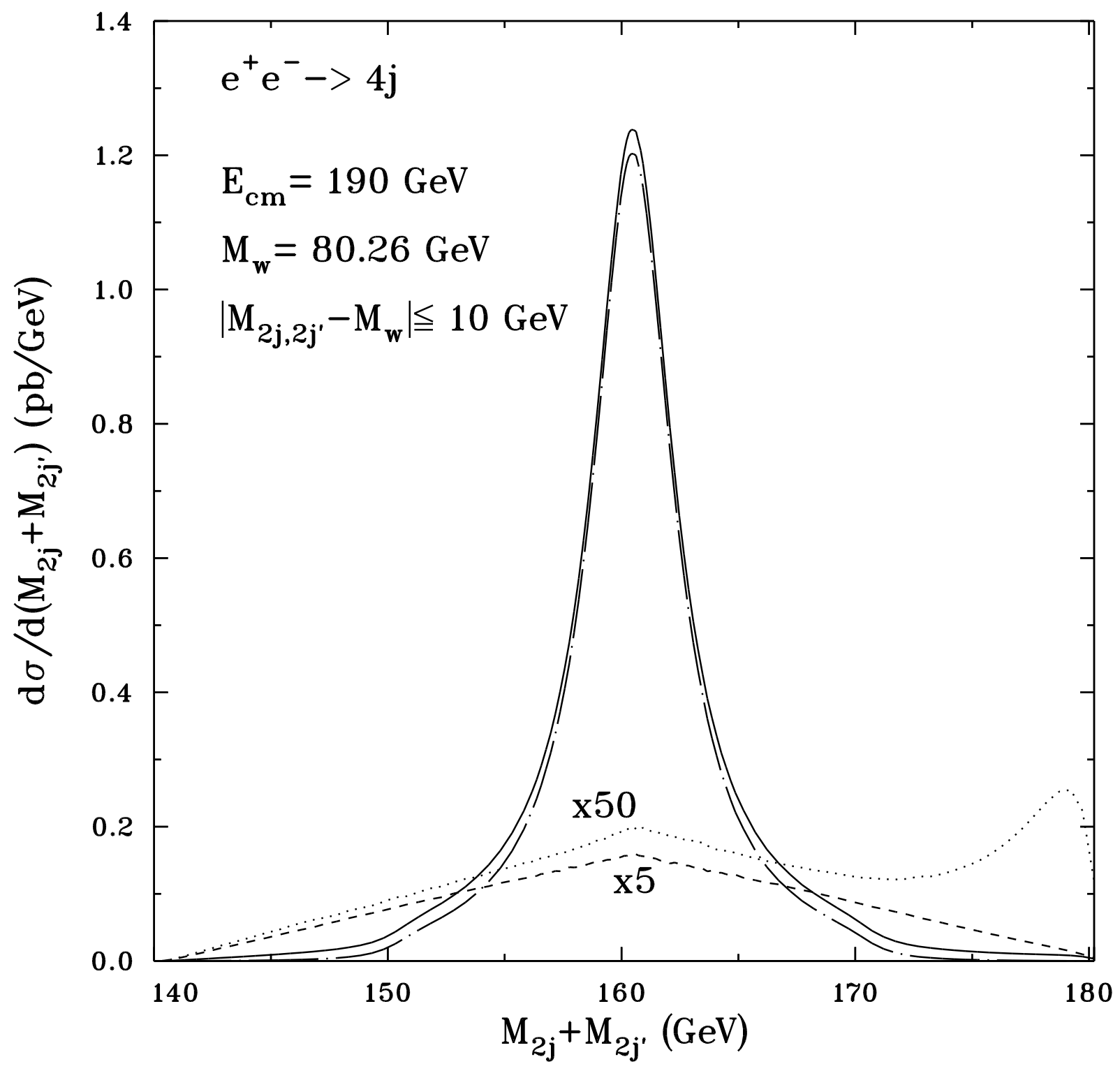

Fig.13- Same distribution as in Fig.11, with $\sqrt{s}=190 \mathrm{GeV}$. 